\title{
F-TOUCH Sensor: Concurrent Geometry Per- ception and Multi-axis Force Measurement
}

\author{
Wanlin $\mathrm{Li}^{1}$, Akram Alomainy ${ }^{2}$, Ivan Vitanov ${ }^{1}$, Yohan $\mathrm{Noh}^{3}$, Peng $\mathrm{Qi}^{4}$ and Kaspar Althoefer ${ }^{1}$
}

\begin{abstract}
Force and tactile sensing has experienced a surge of interest over recent decades, as it conveys a range of information through physical interaction. Tactile sensors aim to obtain tactile information (including pressure, texture etc.). However, current tactile sensors have difficulties in accurately acquiring force signals with regards to magnitude and direction. This is because tactile sensors such as the GelSight sensor estimate shear forces from discrete markers embedded in a compliant sensor interface, employing image processing techniques - the resultant force errors are sizeable. This paper presents a novel design for a force/tactile sensor, namely the F-TOUCH (Force and Tactile Optically Unified Coherent Haptics) sensor, representing an advancement on current vision-based tactile sensors. In addition to acquiring geometric features at a high spatial resolution, our sensor incorporates a number of deformable structural elements allowing us to measure translational and rotational force and torque along six axes with high accuracy. The proposed sensor contains three key components: a coated elastomer layer acting as the compliant sensing medium, spring mechanisms acting as deformable structural elements, and a camera for image capture. The camera records the deformation of the structural elements as well as the distortion of the compliant sensing medium, concurrently acquiring force and tactile information. The sensor is calibrated with the use of a commercial ATI force sensor. An experimental study shows that the F-TOUCH sensor outperforms the GelSight sensor with regard to its capabilities to sense force signals and capturing the geometry of the contacted object.
\end{abstract}

Index Terms-Tactile sensing; force/torque sensor; contact sensing; vision; image processing; robotics

\section{INTRODUCTION}

$\mathrm{F}$ orce and tactile sensing has garnered much research interest over past decades, for it admits of information gathering through direct physical contact between a sensing device and

This work was supported by the EPSRC National Centre for Nuclear Robotics project (EP/R02572X/1), the Innovate UK WormBot project (104059), the CCF-Tencent Open Fund (RAGR20190120), the Open Research Fund (MSV201916) of the State Key Laboratory of Mechanical System and Vibration, Shanghai Jiaotong University, and Zhejiang Lab's International Talent Fund for Young Professionals.

${ }^{1}$ Wanlin Li, Ivan Vitanov and Kaspar Althoefer are with the Centre for Advanced Robotics @ Queen Mary (ARQ), Queen Mary University of London, Mile End Road, London, E1 4NS, United Kingdom (phone: +44 (0)20 7882 3419; e-mail: k.althoefer@qmul.ac.uk).

${ }^{2}$ Akram Alomainy is with the School of Electronic Engineering and Computer Science (EECS), Queen Mary University of London, Mile End Road, London, E1 4NS, United Kingdom (e-mail: a.alomainy@qmul.ac.uk).

${ }^{3}$ Yohan Noh is with Department of Mechanical and Aerospace Engineering at Brunel University London, Kingston Lane, Uxbridge, UB8 3PH, United Kingdom (e-mail: yohan.noh@brunel.ac.uk).

${ }^{4}$ Peng Qi is with Department of Control Science and Engineering, College of Electronics and Information Engineering, Tongji University, No. 1239, Siping Road, Shanghai 200092, China (e-mail: pqi@tongji.edu.cn). the contacted objects. The application of force and tactile sensing with respect to a robot's end effector is vital to achieving next-level grasping and manipulation performance. A proliferation of new sensor designs combining force and tactile measurement has, over recent years, brought this prospect a step closer to reality.

A number of theoretical models of artificial force and tactile sensing systems have been put forward, chief amongst these being that proposed by Howe [36]. It features different sensors being utilized and working together as an integrated whole to perform force and tactile sensing. Included are force sensors mounted between a robot's fingertip and joint, and tactile sensors at the fingertip. Acquired force information includes multi-axis force/torque $(\mathrm{F} / \mathrm{T})$ components measured along the $x$-, $y$-, and $z$-axes; whereas tactile information includes mechanical stimulus detection of contact, slip and pressure.
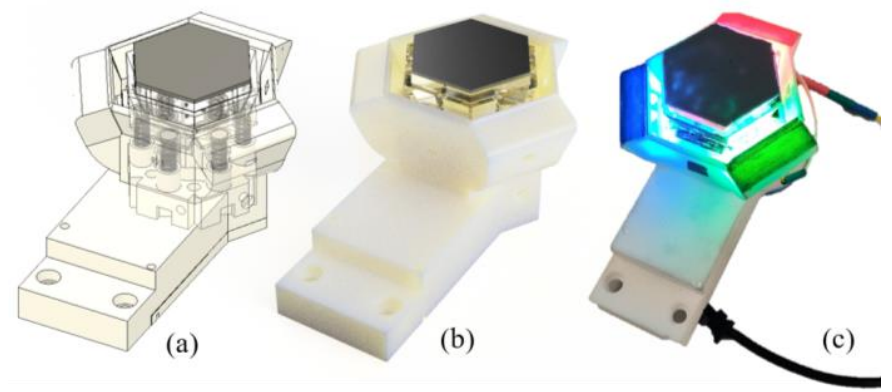

Fig. 1. The proposed F-TOUCH sensor. From left to right are (a) Computer-Aided Design (CAD) view of the sensor, where an internal spring-mechanism structure with three markers is used to measure six-axis force/torque components. (b) Rendering model of the sensor. (c) The manufactured prototype of the sensor. The internal RGB LED light arrays and the webcam are powered via two USB cables, respectively. Both USB cables are connected to a PC. Our sensor is inspired by the fingertip GelSight sensor.

Force sensors have seen extensive development over the past seven decades [37], and their capacity for multi-dimensional force/torque measurement has made them very attractive across a broad swathe of robotic research. Various means of developing multi-axis force sensors have been proposed, including strain sensing technology [38, 39], cross-beam structure [40], Stewart platform [41], and optics [42]. Salient criteria to arrive at a desired force sensor design include considerations of accuracy, robustness, and reliability. Strain gauges were among the earliest and most commonly used technologies, and they have dominated the strain measurement and weighing industry. The ATI multi-axis force/torque sensor [43] uses silicon strain-gauge sensing technology to attain high sensitivity and repeatability and is one of the best-known force sensors currently on the market. Optical-based force sensors have also 
developed rapidly in recent years; a typical example is the OnRobot HEX force/torque sensor (formerly known as the OptoForce sensor) [44]. Other than commercial sensors, many in-lab optical-based force sensors [45-47] have been proposed which make use of optoelectrical components, optical fibers or cameras.

Tactile sensors have drawn interest for yet another reason: they are regarded as the future of robotic grasping [48]. As tactile information has become a key sensing modality, various technologies have been investigated to develop sensors [1-3] of this type. However, current tactile sensing devices are still insufficiently mature due to the inherent complexity of understanding the sense of human touch and its replication in hardware. Many tactile sensors for robots are biologically inspired [4-5], where the aim is to imitate human ability to obtain tactile feedback. Among the different methods that have been applied towards realizing tactile sensors are ones that rely on resistance [6], capacitance [35], piezoelectricity [7], organic field-effect transistors (OFETs) [8], optics [9], and magnetics [10]. Most of the tactile sensors used on robots are designed for fitting onto fingertip-shaped appendages or gripper end-effectors [11-12], while others are designed to be mounted on the body of a robot [13]. The latter have a larger sensing area and are mostly used for contact and collision detection during robot motion.

There are major challenges [28] in developing a desired artificial force and tactile sensor. Firstly, the sensor needs to be small in order to be of a size compatible with standard robots. Secondly, it should at all times provide real-time force and tactile feedback with good signal quality. Thirdly, the sensor should be reproducible, so as to enable other researchers to duplicate it. Fourthly, it ought to be able to measure normal force as well as shear force and torque. Finally, the sensor should be able to handle multi-point touch, not only single-point, with an evenly distributed contact area where each local tactile element ideally has the same characteristics.

In amongst the myriad different sensing technologies, spanning both force and tactile sensors, optical sensors using cameras have a lot to recommend them. This type of sensor can provide a high spatial resolution and, crucially, can sense multiple modalities (such as contact force, contact surface geometry, and hardness) from analyzing a single captured image [14-19]. Vision-based tactile sensors usually use soft and deformable materials as the sensing medium. Deformation of the material can be directly monitored through a camera or by observing changes to certain patterns on the sensing medium to indirectly acquire tactile information. Elastomeric materials offer compliance [52], which gives the tactile sensor the ability to adapt to curved or irregular surfaces during manipulation of objects. For instance, a compliant tactile sensor mounted on a fingertip or end-effector of a robot can assist with grasping different types of objects. Hence, a number of optical tactile sensors leverage deformation and compliance. TacTip [20] is composed of a silicone outer skin with inward facing papilla or nodule pins. Papilla pins on the inside of the sensor's skin are tracked by a camera, and the movements of the pins under external contact are converted into pressure. The dome-shaped three-axis fingertip tactile sensor [21] is capable of measuring normal force and shear force, detected via the deformation and displacement of rubber-made conical feelers attached to the sensing medium. FingerVision [22] is a vision-based tactile sensor that uses a transparent skin for robotic fingers and utilizes a camera to track markers affixed to the skin. This device provides contact force/torque estimates as well as slip detection. Another vision-based tactile sensor [23] has been developed with twin layers of markers inserted into a rubber covering. It is a large, flat sensor and uses a simplified mechanical model to calculate normal and shear forces during point contact. The authors have also proposed a scaled-down variant of their sensor to fit a robot fingertip [24]. GelSight [25] uses a deformable elastomer piece as the medium of contact and an embedded camera is placed at the foot of the sensor to capture the deformation of the elastomer surface. The initial GelSight prototype was presented in [26], without markers on the elastomer. The high-resolution 3D geometry of the contact surface can be reconstructed from the camera images based on the photometric stereo technique. A later version of the sensor [27] features small black markers painted over the entire sensing medium. The motion of the markers provides information about both normal and shear forces. The GelSight sensor can easily capture the detailed shape and texture of the contact object, which makes it useful for material recognition. Nevertheless, the device has limitations; for example, the sensor cannot measure normal force, shear force and torque as accurately as a stand-alone force sensor, since it is difficult to extract the force-related information from the GelSight images due to the presence of numerous markers merging with the tactile information in the image.

Our principal contribution in this work is to match the high fidelity of stand-alone force and tactile sensors with the use of a single, integrated sensor. Seeing as a force sensor cannot measure tactile information, and a tactile sensor cannot accurately measure force information, we require both modalities to co-exist within the same sensing unit. At the same time, premising data acquisition on a single optical transducer (i.e. monocular camera) shared across the two modalities affords us a high level of integration. The use of a unitary shared transducer equates to a reduced hardware footprint for the same proximate data throughput and thereby to increased resource efficiency. Economizing on hardware resources has the ancillary benefit of increased portability of the sensor, such that it can more easily and readily be mounted onto an end effector for instance. We employ a spring-mechanism structure (coupled to a triad of visible markers) beneath the elastomer layer for force sensing, whereby six-axis force/torque components are measured through the corresponding motion and area changes of each marker. The elastomer, meanwhile, conveys tactile geometry information.

This paper is structured as follows. We present the development of the proposed sensing device (Fig. 1) in Section II. Calibration experiments are introduced in Section III. Discussion relating to sensor accuracy, hysteresis and overall performance are presented in Section IV. Section V concludes the paper. 


\section{F-TOUCH SENSOR DESIGN}

A functional tactile sensor should be small in volume in order to be compatible with a robotic hand or gripper. At the same time, it is important that it provides real-time tactile feedback to assist the robot. The fingertip GelSight sensor is a good example of a vision-based tactile sensor that embodies these characteristics. As described in Section I, the chief advantage of its design is the size reduction relative to the original prototype [29], i.e. into a miniaturized form factor [30] to fit a jawed gripper. Equally, the GelSight sensor obtains the geometry of the contact object together with the contact force, which achieves the integration of multiple modalities within one sensing device. However, a large number of black markers are directly painted onto a transparent elastomeric layer (covered with a reflective-coating membrane). Their intended use is to enable the acquisition of force information; however, this leads to the merging of force and tactile information, which meets the requirement of perceiving multiple modalities, but makes it difficult to separate one information source from the other. This is even more of a hindrance when it comes to sensor calibration, especially for the force/torque modality, as it is influenced by the contact geometry during measurement acquisition [31].

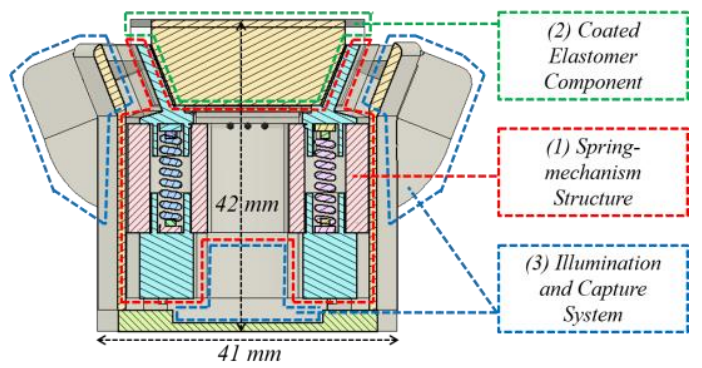

Fig. 2. Schematic of the design of the proposed sensor. Three parts are needed for the device assembly; (1) the spring-mechanism structure is a six-axis platform that holds the (2) coated elastomer component acting as the sensing medium. A camera at the bottom concurrently captures the marker movements and area changes within the spring-mechanism structure, as well as the deformation of the elastomer surface illuminated by internal LEDs. The camera and the LEDs make up the (3) capture and illumination system.

We propose the F-TOUCH (Force and Tactile Optically Unified Coherent Haptics) sensor. It takes inspiration from the GelSight tactile sensor [31]. However, the F-TOUCH sensor is endowed with an enhanced force/torque measurement capability by virtue of an integrated structure consisting of six deformable elements (here: springs). As shown in Fig. 2, the proposed vision-based sensor is composed of three main parts: (1) spring-mechanism structure; (2) coated elastomer component; and (3) illumination and capture system.

\section{A. Spring-mechanism Structure}

The spring-mechanism structure is critical to the proposed sensor design. This force-related structure provides six degrees of freedom (allowing six-axis force/torque measurements) and is placed inside the sensor body. Having more degrees of freedom, the proposed sensor can compliantly adapt to different types of contact conditions. The spring-mechanism structure is composed of six components (as shown in Fig. 3), which can be subdivided into three categories: the upper assembly, the mid-section and the lower assembly.

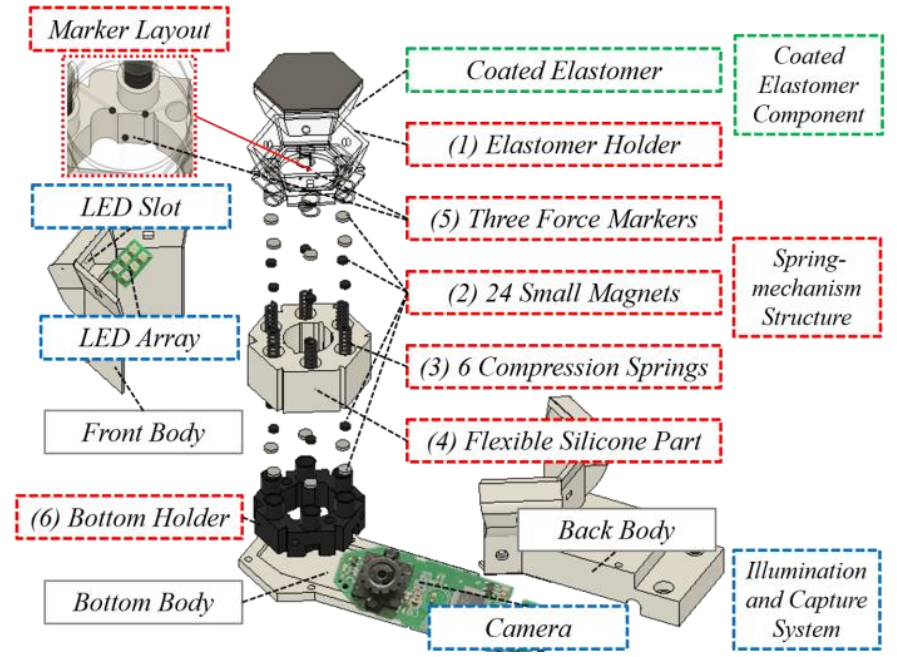

Fig. 3. Exploded view of the proposed sensor's spring-mechanism structure. Six parts (1)-(6) make up the structure: namely, the upper assembly, mid-section and lower assembly: (1) elastomer holder and (5) black markers compose the upper assembly; (3) 6 springs, (2) 24 magnets and (4) a flexible silicone compound compose the mid-section of the structure; (6) bottom holder composes the lower assembly.

Seen from the exploded view in Fig. 3, the upper assembly consists of the elastomer holder (1) and the black markers (5). The elastomer holder is a transparent hollow hexagonal tray which contains the coated elastomer layer (more details are provided in the next sub-section); a 1-mm-thick clear acrylic sheet is laser-cut to fill out the middle of the tray. Three markers are situated in the middle of the elastomer holder, held in place by three thin supports. The elastomer holder together with the three markers is 3D-printed as one contiguous ensemble. It uses clear material $\left(\right.$ VeroClear $^{\circledR}$ ) to facilitate LED illumination. The markers are drawn in black with an acrylic paint pen after fabrication. At the base of the elastomer holder, six 4-mm-diameter magnets are glued inside six reserved apertures to allow connection with the springs of the mid-section. In the mid-section, six compression springs (3) (with a 3-mm-diameter magnet glued to either end of each spring, for a total of 12 magnets) are passed through a white silicone-made flexible compound (4) to connect the upper assembly and the lower assembly. The compression springs are of $15 \mathrm{~mm}$ length, $4 \mathrm{~mm}$ in diameter, and of $0.5 \mathrm{~mm}$ wire diameter. The connections rely on magnetic attraction (2), which provides a very stable hold. A flexible, hollow silicone component made from an Ecoflex 0030 platinum-cure silicone rubber compound (see Table I) covers the six springs, for a stable connection between the upper assembly and the lower assembly. Also, it enables a wider range of force/torque measurement. The lower assembly consists of the 3D-printed bottom holder (in nylon material) (6), where another set of six 4-mm-diameter magnets are glued in, in the same way as on the upper assembly's surface plane. The lower assembly cradles the camera lens; the hollow of the bottom holder frames the camera lens and keeps it trained on both the black markers and the coated elastomer surface. The proposed spring-mechanism can sustain a maximum normal load of up to $8 \mathrm{~N}$ during testing.

We can view the movements of the three black circular markers as reciprocal with the compression of the six springs. 
We estimate the six-axis force/torque components by observing the markers' movements and their area changes, as relayed by the camera. When an external force is applied on the elastomer, the exerted force/torque components will cause a compression of the spring-mechanism structure. Because of the connectivity between elastomer and spring structure, these movements, as well as the areas of the three circular markers within the spring structure, will be coupled to those of the sensing medium. A more detailed explanation will be provided in the next section. It is worth noting that the spring-mechanism structure is designed to measure six-axis force/torque information, as the coated elastomer has a relatively high rigidity compared to the low-spring-constant compression springs and soft flexible silicone compound. The state of the captured markers is directly related to the overall force/torque components exerted upon the sensing medium.

TABLE I

SILICONE MATERIAL PROPERTIES

\begin{tabular}{|c|c|c|c|}
\hline \multirow{2}{*}{ Property } & \multicolumn{3}{|c|}{ Platinum Cure Silicone } \\
\cline { 2 - 4 } & Ecoflex 0030 & Solaris & Psycho Paint \\
\hline Mix Ratio By Weight & $1 \mathrm{~A}: 1 \mathrm{~B}$ & $1 \mathrm{~A}: 1 \mathrm{~B}$ & $1 \mathrm{~A}: 1 \mathrm{~B}$ \\
\hline Elongation at Break & $900 \%$ & $290 \%$ & $1,000 \%$ \\
\hline Shore Hardness & $00-30$ & $15 \mathrm{~A}$ & Not Given \\
\hline Color & Translucent & Clear & Translucent \\
\hline
\end{tabular}

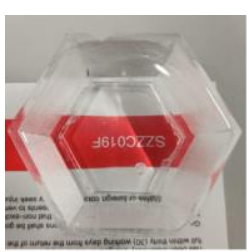

(a)

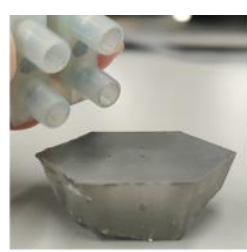

(b)

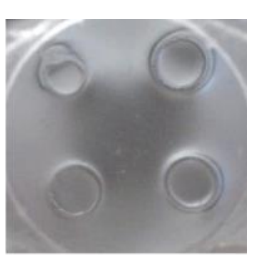

(c)
Fig. 4. Manufactured silicone elastomer component. (a) The transparent silicone base placed on a piece of paper; (b) An object with four cylindrical legs is pressed against the coated elastomer component; (c) Camera view from the underside of the coated elastomer capturing the distortion of the elastomer's coated surface.

\section{B. Coated Elastomer Component}

The nature of the elastomer component largely determines the sensor's performance. It is made up of two parts: the elastomeric base and coating membrane. To create a suitable elastomeric base for tactile sensing, the materials used need to be optically clear, soft yet robust, and readily available. $\mathrm{Li}$ and Adelson[32] recommend a Shore hardness range for the elastomeric base that is between Shore A values 5 and 20. Following this recommendation, we use Solaris (part A and part B) with Shore A 15 (see Table I) and Slacker (used to increase softness) from vendor Smooth-on ${ }^{\circledR}$ to produce the transparent elastomeric base. A ratio of 1:1:1 for each component has proven to be ideal for making an elastomeric base with the appropriate hardness [31]. As for the coating membrane, it is a very thin but reflective layer that covers the upper surface of the transparent elastomer. The layer needs to be thin, uniform and smooth. A matte coating, made by mixing silicone and fine metallic powders [51], is preferable for tactile sensing, as it is effective at revealing detailed shapes on the contact surface. We use a platinum-cure silicone named Psycho Paint (see Table I), and we disperse aluminum powder into a silicone paint base for the coating on top of the elastomer base. In practice, an elastomeric component fabricated in this way is able to sustain a moderate force/torque exertion while revealing the fine geometry of the contact object's surface (see Fig. 4), which is comparable to the GelSight sensor's elastomer.

\section{Illumination and Capture System}

The illumination and capture system provides the physical basis for the disaggregation of force-related information from the tactile image. We use RGB SMD LEDs (Osram ${ }^{\circledR}$ Opto Power TOPLED Lens) within the sensor body for internal illumination. The reason for choosing RGB color is based on the fact that the initial GelSight sensor [29] used monochrome illumination but later switched over to RGB illumination for surface reconstruction [25]. In our view, RGB illumination will be more effective in future tactile perception, in particular for geometry reconstruction and recognition. Besides, it is also easy to extract the black markers from the RGB background via simple thresholding, which enables real-time force and tactile data acquisition. Fig. 5 presents the configuration of the proposed illumination and capture system: three PCBs, each with six LEDs (arranged into $2 \times 3$ arrays) of the same color, are installed within predesigned slots on the sensor body. As described above, we use a clear elastomer holder to contain the transparent elastomer component, as the tray can homogenize the LEDs' light while allowing a high transmission rate. An off-the-shelf webcam (Logitech ${ }^{\circledR}$ C920, manual focus, resolution of 640x480 pixels, 30 frames per second) is placed about $15 \mathrm{~mm}$ away beneath the elastomer holder. We manually set the focal length so that the camera can capture a high-resolution tactile image, while concurrently capturing the three black circular markers - for acquisition of force-related information.

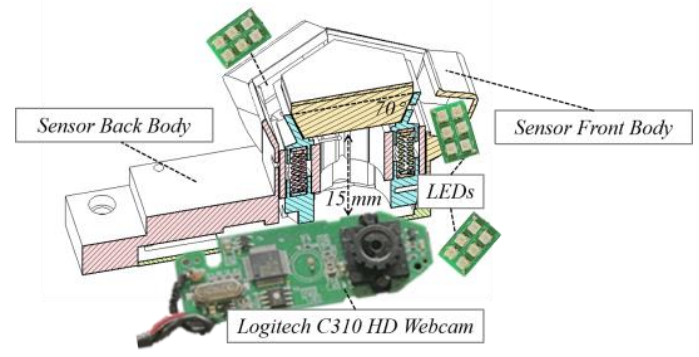

Fig. 5. Illumination and capture system within F-TOUCH. Three RGB LED arrays are employed for internal illumination and an off-the-shelf camera is used for image capturing.

\section{Sensor Measurement Methodology}

The key contribution of the proposed sensor is the capability to concurrently and effectively perceive dual modalities. In F-TOUCH, a unitary force and tactile sensing capability is integrated within a single sensor housing, with signal acquisition being performed by a single monocular camera acting as the transducer.

As elaborated in Section II, the measurement of the net force/torque components is based on the position and area changes of the three black markers contained within the elastomer holder (see Fig. 6(a)). Since the elastomer component is glued inside the elastomer holder, we can treat both parts as 
constituting an integrated whole. Hence, the movement of the elastomer holder can be seen to represent the net external force/torque applied upon the sensing medium. When no contact occurs, the camera captures the illuminated elastomer surface and the markers as shown in Fig. 6(b). The next step is to segment the force-related information from the tactile image background. Since we use RGB light as internal illumination, and the markers are painted black, we convert the raw captured image into grayscale and apply a Gaussian filter for smoothing. We then set a threshold and reverse the color back to the filtered grayscale image to obtain a binary image, as shown in Fig. 6(c).

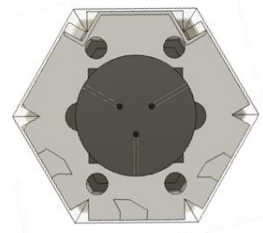

(a)

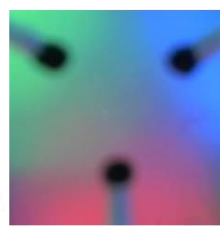

(b)

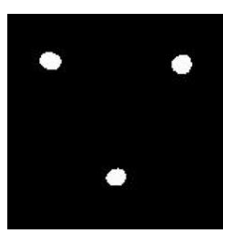

(c)

Fig. 6. Extracting force markers from the tactile image. (a) Bottom view of the CAD spring-mechanism structure, where force markers are circularly attached beneath the coated elastomeric component. (b) The raw captured image from the camera. (c) The binary image after thresholding.

(1) $+\mathrm{Fx}-\mathrm{Ty}$

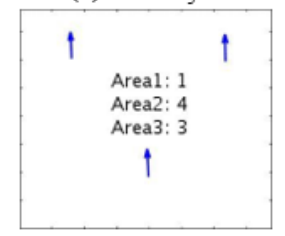

(4) $-\mathrm{Fx}-\mathrm{Fy}-\mathrm{Tx}+\mathrm{Ty}$

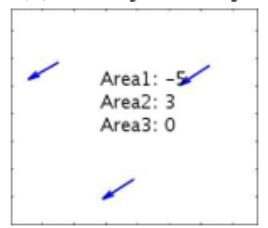

(7) $-\mathrm{Fz}-\mathrm{Tx}-\mathrm{Ty}$

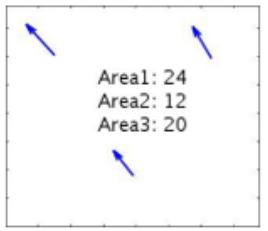

Fig. 7. Responses of the F-Touch sensor under different force/torque loads. The sensor responses are in a form of depicting the motion of three marker centroids as a vector plot, together with the corresponding marker area changes. Each marker's movement vector is scaled for better comprehension of the motions. Nine scenarios of force/torque loading conditions are presented: (1) Shear force $+F x$ and torque - $T y$ are applied; (2) Shear force $-F y$ and torque - $T x$ are applied; (3) Shear force $-F x+F y$ and torque $+T x+T y$ are applied; (4) Shear force $-F x$ $-F y$ and torque $-T x+T y$ are applied; (5) Normal force $-F z$ and torque $+T x$ are applied; (6) Normal force $-F z$ and torque $+T y$ are applied; (7) Normal force $-F z$ and torque $-T x-T y$ are applied; (8) Torque $+T z$ is applied; (9) Torque $-T z$ is applied.

As described in the above section, we assume that the centroids of each of the circular markers are within a plane that is in parallel to the elastomer's surface plane. For example, a normal force acting on the center point of the sensing medium leads to a height difference of the markers' plane with respect to the reference (unloaded) state. Effectively, the markers come closer to the camera and thus appear larger in the camera images. In other words, a change in distance between a marker and the camera leads to a change in the area covered by the marker's camera image. When normal force and shear force are concurrently applied to the sensing medium (the elastomer plane), the distance between the markers' plane and the camera changes (and the markers' plane experiences a tilt), resulting in a marker area change as well as a marker movement across the camera image. Our aim is to track the movement of the three markers along with their area change, thus we detect and label the markers in the binary image (see Fig. 6(c)). We then calculate both centroid positions and areas of the three labelled markers in the image. We set the original $x, y$ coordinate values of each marker in unloaded condition as $x_{i}(0)$ and $y_{i}(0)$ and the corresponding area as $a_{i}(0)$ where $i$ is the label index $i=$ $1,2,3$. During the contact, we calculate the changes of both the markers' centroid positions (all displacements are in cartesian coordinates $\Delta x_{i}(j)=x_{i}(j)-x_{i}(0), \Delta y_{i}(j)=y_{i}(j)-y_{i}(0)$ and the areas $\Delta a_{i}(j)=a_{i}(j)-a_{i}(0)$ for each frame $j$, where $(i=1,2,3)$.

We then visualize the markers' centroid motions as vector plots, along with the respective area changes. Fig. 7 depicts the F-TOUCH sensor responses in the form of marker motions and area changes under nine different loading conditions, where different force/torques are applied using the setup in Fig. 9. Having obtained values for the nine variables $\Delta x_{1} \Delta y_{1} \Delta a_{1} \Delta x_{2}$ $\Delta y_{2} \Delta a_{2} \Delta x_{3} \Delta y_{3} \Delta a_{3}$ from three markers, we can proceed to six-axis force/torque calibration of the sensor.

\section{SEnSOR CALIBRATION AND VALIDATION}

The sensor calibration is the process of determining the relationship between the sensor's output variables to the six-axis force and torque input components ( $F x F y F z T x T y T z$ ). There are various studies [49] on force calibration of vision-based tactile sensors. Included are using convolutional neural networks (CNNs), that are useful when dealing with images [31, 50]. However, in our case, there are two sensing modalities (force and tactile) presented in each image, hence we cannot use the exact same method as above. We obtain the numerical force-related numerical values from the image and, given the linearity of the sensor's spring mechanism structure, we apply a conventional force sensor calibration method [42] [33]. The rationale of the calibration process is to calculate the calibration matrix $K$, where the regression coefficients matrix is used to convert the output variables to force and moment loading data. A six-by-nine calibration matrix multiplies the nine-by-one sensor output vector to give the decoupled output force/torque values.

$$
\left[\begin{array}{lllllllll}
k_{11} & k_{12} & k_{13} & k_{14} & k_{15} & k_{16} & k_{17} & k_{18} & k_{19} \\
k_{21} & k_{22} & k_{23} & k_{24} & k_{25} & k_{26} & k_{27} & k_{28} & k_{29} \\
k_{31} & k_{32} & k_{33} & k_{34} & k_{35} & k_{36} & k_{37} & k_{38} & k_{39} \\
k_{41} & k_{42} & k_{43} & k_{44} & k_{45} & k_{46} & k_{47} & k_{48} & k_{49} \\
k_{51} & k_{52} & k_{53} & k_{54} & k_{55} & k_{56} & k_{57} & k_{58} & k_{59} \\
k_{61} & k_{62} & k_{63} & k_{64} & k_{65} & k_{66} & k_{67} & k_{68} & k_{69}
\end{array}\right] \cdot\left[\begin{array}{l}
\Delta x_{1} \\
\Delta y_{1} \\
\Delta a_{1} \\
\Delta x_{2} \\
\Delta y_{2} \\
\Delta a_{2} \\
\Delta x_{3} \\
\Delta y_{3} \\
\Delta a_{3}
\end{array}\right]=\left[\begin{array}{c}
F x \\
F y \\
F z \\
T_{x} \\
T_{y} \\
T_{z}
\end{array}\right]
$$




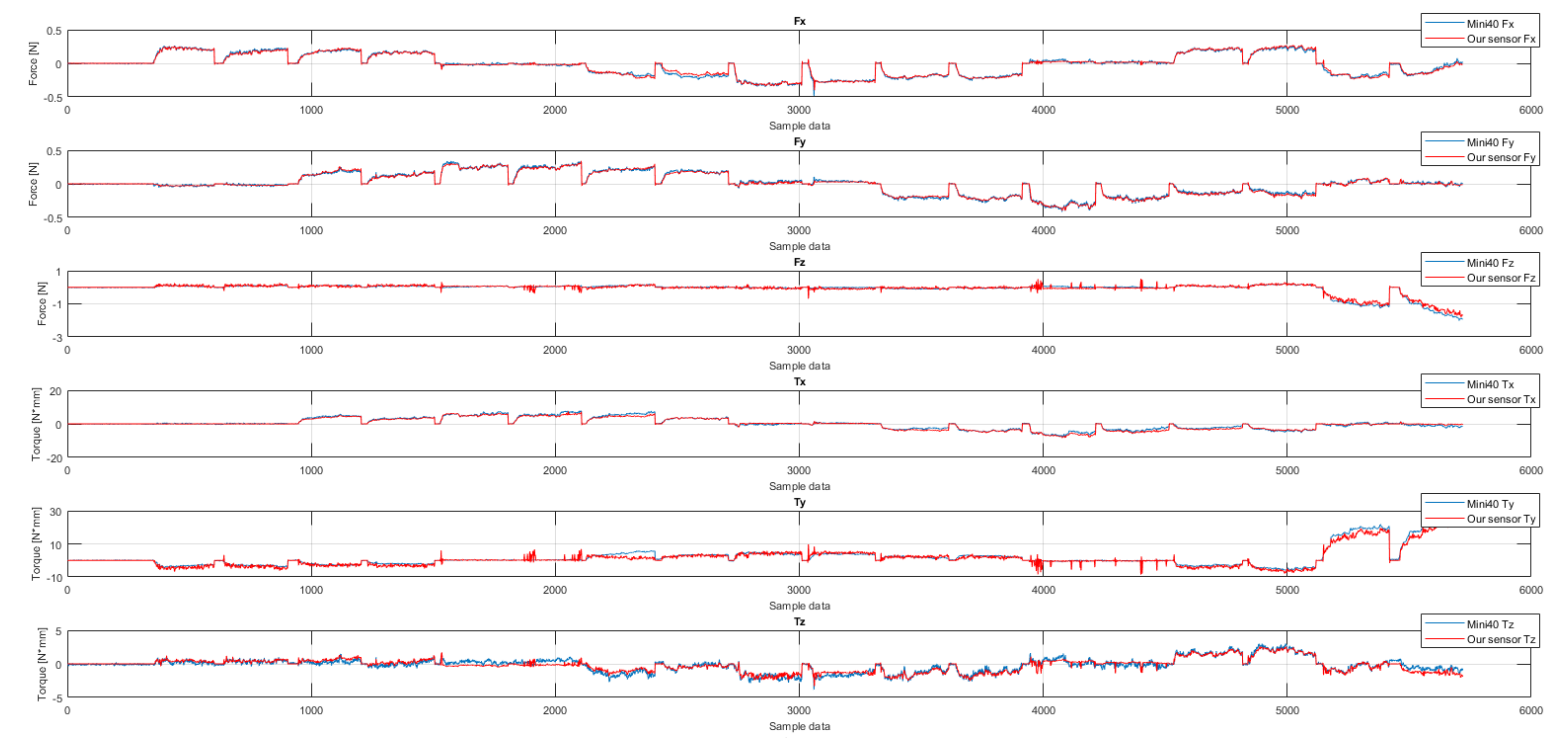

Fig. 8. Comparison of six-axis force/torque performance between ATI $^{\circledR}$ Mini40 sensor and our proposed sensor using a calibration connector.

We set out to calibrate the six-axis force and torque component observations of the F-TOUCH sensor against a high-grade commercial six-axis force/torque sensor $\left(\mathrm{ATI}^{\circledR}\right.$ Mini40 F/T sensor) as a source of reference values [33]. As the sensing medium of the proposed sensor is made of silicone elastomer - a soft material, it is impossible to directly mount the $\mathrm{ATI}^{\circledR}$ sensor atop the elastomer surface. Neither could we directly place the $\mathrm{ATI}^{\circledR}$ sensor underneath our sensor as the torque readings of both sensors would diverge due to the intervening distance between their respective contact surfaces. In order to fulfil the requirements for calibration, a specially designed calibration interface is proposed, as shown in Fig. 9. The commercialized ATI ${ }^{\circledR}$ Mini40 sensor provides the ground-truth force and torque magnitudes, while a force/torque cap (3D-printed using polylactic acid (PLA)) is placed on top of it, and a specially designed connector (3D-printed using acrylonitrile butadiene styrene (ABS)) is attached to its underside. The reverse side of the connector attaches to the elastomer holder of the proposed sensor. The force/torque cap enables different forces and torques to be applied to both sensors through the same plane, as shown in Table II.

TABLE II

FORCE/TORQUE EXERTION CONDITIONS ON THE TIP

\begin{tabular}{|c|c|c|c|}
\hline \multirow{2}{*}{ Applied F/T } & F/T Condition & Applied F/T & F/T Condition \\
\hline 1 & $-F z-T y$ & 2 & $-F z-T x-T y$ \\
\hline 3 & $-F z-T x$ & $(4)$ & $-F z-T x T y$ \\
\hline 5 & $-F z T y$ & 6 & $-F z T x T y$ \\
\hline 7 & $-F z T x$ & 8 & $-F z T x-T y$ \\
\hline 9 & $F y T x$ & 10 & $F x F y T x-T y$ \\
\hline 11 & $F x-T y$ & 12 & $F x-F y-T x-T y$ \\
\hline 13 & $-F y-T x$ & 14 & $-F x-F y-T x T y$ \\
\hline 15 & $-F x T y$ & $(16)$ & $-F x F y T x T y$ \\
\hline 17 & $\pm T z$ & & \\
\hline
\end{tabular}

The connector (shown in Fig. 9(b)) is designed to act as 'connective tissue' between the two sensors. During the calibration process, we replaced the original elastomer component with the specially designed connector, both having the same height. We deliberately chose the connector to be of a white color so that the final binary images of the illuminated markers would be as closely matched as possible while using either the elastomer or the connector.

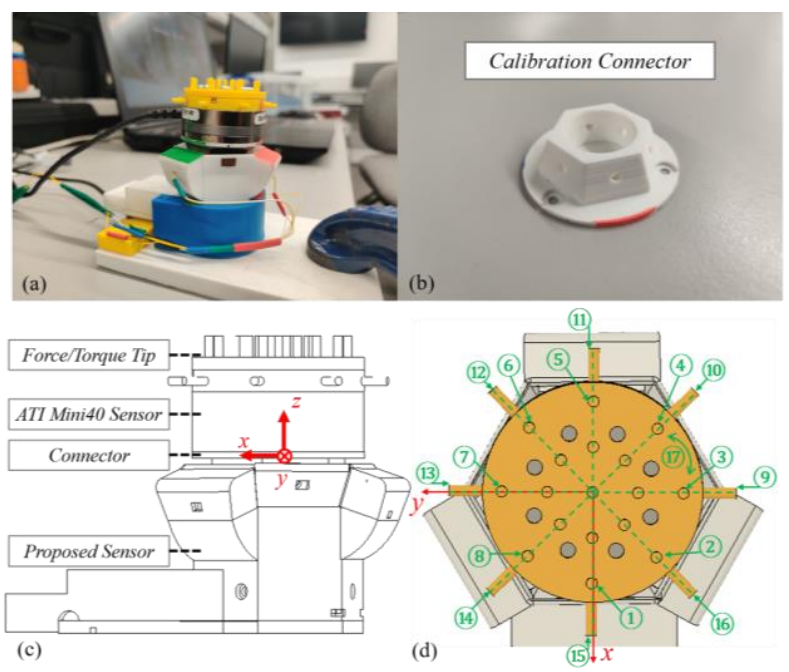

Fig. 9. Calibration of the F-TOUCH sensor, with the aid of a commercial six-axis force/torque sensor $\mathrm{ATI}^{\circledR}$ Mini40 and a specially designed 3D-printed calibration connector. (a) Experimental setup for six-axis force/torque calibration. (b) 3D-printed calibration connector made of ABS material. (c) CAD view of the calibration setup. (d) CAD top-down view of the calibration device for applying different force and torque conditions to both ATI ${ }^{\circledR}$ Mini40 sensor (regarded as the ground truth) and the F-TOUCH sensor. Seventeen loading conditions (see Table II) are applied during the calibration process.

The nine output variables returned by the F-TOUCH captured image data are synchronized at a sampling rate of 30 samples per second with the six-axis force/torque readings from the ATI $^{\circledR}$ Mini 40 sensor under seventeen different loading conditions. We manually exert the force and torque loads on the force/torque cap, as shown in Fig. 9(d). The calibration matrix $K$ is then calculated with the least squares method (LSM) of [34]. 


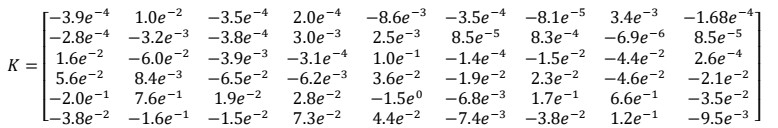

Fig. 8 compares the six-axis force/torque performance between the proposed sensor and the ATI ${ }^{\circledR}$ Mini40 sensor when the connector is used. Due to the use of a camera, the response time of our sensor is $30 \mathrm{~Hz}$, or $33 \mathrm{~ms}$. The RMSE (root mean square error), the corresponding normalized RMSE and the coefficient of determination of fit, $R^{2}$, of F/T components from both our sensor and GelSight sensor are listed in Table III. It can be seen from the table that our sensor exhibits superior force and torque performance over GelSight when comparing with the ground truth measured by the ATI F/T sensor.

TABLE III

PROPOSED ForCE/TORQUE SENSOR CALIBRATION ACCURACY

\begin{tabular}{|c|c|c|c|}
\hline $\begin{array}{c}\text { Force/ } \\
\text { Torque }\end{array}$ & $\begin{array}{c}\text { Range of our } \\
\text { sensor }\end{array}$ & $\begin{array}{c}\text { RMSE, normalized } \\
\text { RMSE and } \\
\text { R-squared in our } \\
\text { sensor }\end{array}$ & $\begin{array}{c}\text { RMSE, normalized } \\
\text { RMSE and } \\
\text { R-squared in Gel- } \\
\text { Sight sensor }\end{array}$ \\
\hline$F x$ & $+/-1 \mathrm{~N}$ & $\begin{array}{c}0.018 \mathrm{~N}(1.8 \%) \\
\mathrm{R}^{2}=0.99\end{array}$ & $\begin{array}{c}0.187 \mathrm{~N}(4.7 \%) \\
\mathrm{R}^{2}=0.94\end{array}$ \\
\hline$F y$ & $+/-1 \mathrm{~N}$ & $\begin{array}{c}0.016 \mathrm{~N}(1.6 \%) \\
\mathrm{R}^{2}=0.99\end{array}$ & $\begin{array}{c}0.162 \mathrm{~N}(4 \%) \\
\mathrm{R}^{2}=0.93\end{array}$ \\
\hline$F z$ & $0-8 \mathrm{~N}$ & $\begin{array}{c}0.094 \mathrm{~N}(1.2 \%) \\
\mathrm{R}^{2}=0.94\end{array}$ & $\begin{array}{c}0.668 \mathrm{~N}(3.3 \%) \\
\mathrm{R}^{2}=0.97\end{array}$ \\
\hline$T x$ & $+/-20 \mathrm{~N} \cdot \mathrm{mm}$ & $\begin{array}{c}0.657 \mathrm{~N} \cdot \mathrm{mm}(3.3 \%) \\
\mathrm{R}^{2}=0.96\end{array}$ & - \\
\hline$T y$ & $+/-20 \mathrm{~N} \cdot \mathrm{mm}$ & $\begin{array}{c}1.566 \mathrm{~N} \cdot \mathrm{mm}(7.8 \%) \\
\mathrm{R}^{2}=0.94\end{array}$ & - \\
\hline$T z$ & $+/-20 \mathrm{~N} \cdot \mathrm{mm}$ & $\begin{array}{c}0.407 \mathrm{~N} \cdot \mathrm{mm}(2 \%) \\
\mathrm{R}^{2}=0.85\end{array}$ & $\begin{array}{c}3.72 \mathrm{~N} \cdot \mathrm{mm}(2.5 \%) \\
\mathrm{R}^{2}=0.84\end{array}$ \\
\hline
\end{tabular}
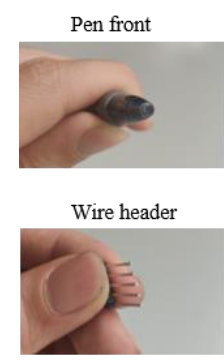

USB header
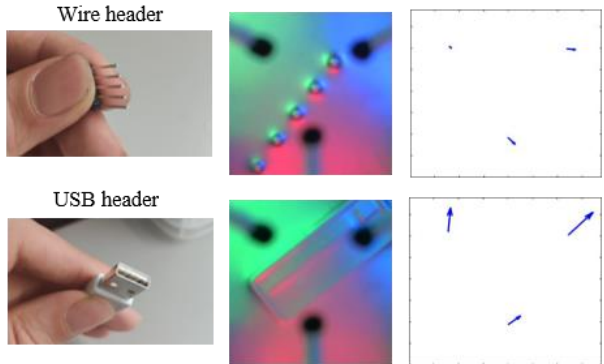

Fig. 10. The F-TOUCH sensor's performance across dual modalities (six-axis force/torque information and contact object's surface geometry). Three different objects are pressed against our sensor's membrane surface. The first row presents the F-TOUCH performance when a pen's front tip is tested; the second row is testing with a wire header; the third row is testing with a USB header.

As the proposed sensor can also perceive the geometry of the contact object's surface, we further explore the proposed sensor's performance by investigating different contact conditions with objects having different surface geometries, as shown in Fig. 10 above, where raw tactile images and six-axis force/torque measurements are presented. In view of the above, we feel justified in concluding that the proposed F-TOUCH sensor has the capability concurrently to perceive two modali- ties: six-axis force/torque components measurement and perception of contact surface geometry, all by processing a single captured image.

\section{DISCUSSION}

The measurement of force/torque components using an elastomer-based tactile sensor is complicated. We have developed a force and tactile sensor architecture that integrates a coated transparent silicone elastomer with a six-degree-of-freedom spring-mechanism force structure. Tracking a trio of markers within the force structure, using a conventional force sensor calibration method, and a specially designed connector and commercial force/torque sensor (ATI ${ }^{\circledR}$ Mini40 sensor), allows six-axis force/torque components to be extracted, calibrated and measured. Moreover, the deformation of the illuminated elastomer surface reflects the contact surface geometry in the same image capture containing the force information; so that observation data for the two modalities can be acquired at the same time with an integrated force and tactile sensing device.

In our experiments, the positions of marker centroids together with marker area changes were shown to effectively represent the net normal force, shear force and torque. With the aid of a commercial $\mathrm{F} / \mathrm{T}$ sensor and a specially designed connector attached to our sensor, a 6-by-9 calibration matrix was computed using the least squares regression method. Future work might explore calibration methods used in tactile sensors.

There remain deviations between the estimated force/torque and ground truth (from the $\mathrm{ATI}^{\circledR}$ Mini40 sensor). A plausible explanation for the discrepancy might run as follows. Since we use both an elastomer component and springs in the sensor structure, hysteresis will inevitably creep in due to the responsiveness of these materials. Thus, the accuracy of the calibration matrix may be affected. Moreover, using a high-quality camera with a higher input rate can reduce image noise. These noise terms can also be improved with a more advanced filtering algorithm. There is always a trade-off between image quality and sensor response rate. When it comes to measuring hysteresis and repeatability, a more complicated testbed architecture is needed. A possible way forward is to introduce a motorized system with a linear guide, so that identical loading conditions can be replicated via the force/torque cap during calibration.

\section{CONCLUSION AND FUTURE WORK}

In this paper we have introduced a unified vision-based sensor, named F-TOUCH (Force and Tactile Optically Unified Coherent Haptics), that can concurrently sense two modalities: six-axis force/torque components and tactile information (in the form of geometry and texture perception) using a single image capturing device. In other words, the F-TOUCH sensor can be regarded as a unitary device combining the capabilities of both a force sensor and a tactile sensor. The proposed sensor is designed to fit robot grippers and takes inspiration from the fingertip GelSight tactile sensor. The novelty and original contribution of the work is in the use of a coated transparent elastomer layer combined with spring-based deformable structural elements to measure tactile and 6-axis force information within a single sensor housing. We are able to extract translational and 
rotational force information (based on the status of the markers integrated with the deformable structural elements) as well as tactile information (based on the deformation of the illuminated elastomer surface) in real-time. Moreover, we have designed a specific implement to assist with the six-axis force/torque calibration of the F-TOUCH sensor. Our experiments show an accuracy of force/torque measurements $(F x, F y, F z, T x, T y, T z$ with RMSE of $0.018 \mathrm{~N}, 0.016 \mathrm{~N}, 0.094 \mathrm{~N}, 0.657 \mathrm{~N} \cdot \mathrm{mm}, 1.566$ $\mathrm{N} \cdot \mathrm{mm}$ and $0.407 \mathrm{~N} \cdot \mathrm{mm}$ respectively) that is superior to the GelSight sensor's force/torque sensing performance.

In future work, we plan to explore tactile image analysis towards surface reconstruction and pressure distribution. Texture classification is also planned.

\section{REFERENCES}

[1] B. Ward-Cherrier, N. Pestell, L. Cramphorn, B. Winstone, M. E. Giannaccini, J. Rossiter, and N. F. Lepora, "The TacTip Family: Soft Optical Tactile Sensors with 3D-Printed Biomimetic Morphologies," in Soft Robot., Jan. 2018.

[2] D. Ma, E. Donlon, S. Dong and A. Rodriguez, "Dense Tactile Force Estimation using GelSlim and inverse FEM," in 2019 International Conference on Robotics and Automation (ICRA), Montreal, QC, Canada, 2019, pp. 5418-5424.

[3] R. A. Romeo and L. Zollo, "Methods and Sensors for Slip Detection in Robotics: A Survey," in IEEE Access, vol. 8, pp. 73027-73050, 2020

[4] J. W. James, N. Pestell and N. F. Lepora, "Slip Detection With a Biomimetic Tactile Sensor," in IEEE Robotics and Automation Letters, vol. 3, no. 4, pp. 3340-3346, Oct. 2018.

[5] W. Li, J. Konstantinova, Y. Noh, Z. Ma, A. Alomainy and K. Althoefer, "An Elastomer-based Flexible Optical Force and Tactile Sensor," in 2019 2nd IEEE International Conference on Soft Robotics (RoboSoft), Seoul, Korea (South), 2019, pp. 361-366.

[6] K. Bae, S. Pyo and J. Kim, "Crosstalk-Free Mesh-Embedded Tactile Sensor Array with Electrically Isolated Sensing Cells," in 2020 IEEE 33rd International Conference on Micro Electro Mechanical Systems (MEMS), Vancouver, BC, Canada, 2020, pp. 673-676.

[7] A. Ke et al., "Fingertip Tactile Sensor With Single Sensing Element Based on FSR and PVDF," in IEEE Sensors Journal, vol. 19, no. 23, pp. 11100-11112, 1 Dec.1, 2019

[8] X. Wu and J. Huang, "Array of Organic Field-Effect Transistor for Advanced Sensing," in IEEE Journal on Emerging and Selected Topics in Circuits and Systems, vol. 7, no. 1, pp. 92-101, March 2017.

[9] J. Konstantinova, A. Stilli and K. Althoefer, "Fingertip Fiber Optical Tactile Array with Two-Level Spring Structure," in Sensors 17(10), 2337, 2017

[10] A. C. Holgado et al., "An Adjustable Force Sensitive Sensor with an Electromagnet for a Soft, Distributed, Digital 3-axis Skin Sensor," in 2018 IEEE/RSJ International Conference on Intelligent Robots and Systems (IROS), Madrid, 2018, pp. 2582-2588.

[11] Liu, Hongbin, et al. "Finger contact sensing and the application in dexterous hand manipulation, " in Autonomous Robots 39.1 (2015): 25-41, 2015.

[12] E. Donlon, S. Dong, M. Liu, J. Li, E. Adelson and A. Rodriguez, "GelSlim: A High-Resolution, Compact, Robust, and Calibrated Tactile-sensing Finger," in 2018 IEEE/RSJ International Conference on Intelligent Robots and Systems (IROS), Madrid, 2018, pp. 1927-1934.

[13] T. Le, P. Maiolino, F. Mastrogiovanni and G. Cannata, "Skinning a Robot: Design Methodologies for Large-Scale Robot Skin," in IEEE Robotics \& Automation Magazine, vol. 23, no. 4, pp. 150-159, Dec. 2016.

[14] J. L. Schneiter and T. B. Sheridan, "An optical tactile sensor for manipulators," in Robotics Computer-Integrated Manufacturing, vol. 1, pp. 65-71, 1984.

[15] S. Begej, "Planar and finger-shaped optical tactile sensors for robotic applications," in IEEE Journal on Robotics and Automation, vol. 4, no. 5, pp. 472-484, Oct. 1988.

[16] M.K. Johnson, et al. "Microgeometry capture using an elastomeric sensor." in ACM Transactions on Graphics (TOG) 30.4 (2011): 1-8, 2011.

[17] N. Pestell, J. Lloyd, J. Rossiter and N. F. Lepora, "Dual-Modal Tactile Perception and Exploration," in IEEE Robotics and Automation Letters, vol. 3, no. 2, pp. 1033-1040, April 2018
[18] W Li, J. Konstantinova, A. Alomainly and K. Althoefer, "Elastomer Based Touch Sensor: Visualization of Tactile Pressure Distribution," in Annual Conference Towards Autonomous Robotic Systems (TAROS), London, 2019, pp. 87-98.

[19] T. Sakuma, F. Von Drigalski, M. Ding, J. Takamatsu and T. Ogasawara, "A Universal Gripper Using Optical Sensing to Acquire Tactile Information and Membrane Deformation," in 2018 IEEE/RSJ International Conference on Intelligent Robots and Systems (IROS), Madrid, 2018, pp. $1-9$.

[20] B. Winstone, G. Griffiths, T. Pipe and J. Rossiter, "TACTIP-Tactile fingertip device texture analysis through optical tracking of skin features," in Biomimetic Biohybrid Systems, pp. 323-334, 2013.

[21] M. Ohka, Y. Mitsuya, I. Higashioka and H. Kabeshita, "An experimental optical three-axis tactile sensor for micro-robots," in Robotica 23, no. 4, pp. 457-465, 2005.

[22] A. Yamaguchi and C. G. Atkeson, "Implementing tactile behaviors using FingerVision," in 2017 IEEE-RAS 17th International Conference on Humanoid Robotics (Humanoids), Birmingham, 2017, pp. 241-248.

[23] K. Kamiyama, K. Vlack, T. Mizota, H. Kajimoto, K. Kawakami and S. Tachi, "Vision-based sensor for real-time measuring of surface traction fields," in IEEE Computer Graphics and Applications, vol. 25, no. 1, pp. 68-75, Jan.-Feb. 2005.

[24] K. Sato, K. Kamiyama, N. Kawakami, and S. Tachi, "Finger-shaped GelForce: sensor for measuring surface traction fields for robotic hand," in IEEE Transactions on Haptics, 3(1):37-47, Jan. 2010.

[25] S. Dong, W. Yuan and E. H. Adelson, "Improved GelSight tactile sensor for measuring geometry and slip," in 2017 IEEE/RSJ International Conference on Intelligent Robots and Systems (IROS), Vancouver, BC, 2017, pp. 137-144.

[26] M. K. Johnson and E. H. Adelson, "Retrographic sensing for the measurement of surface texture and shape," in 2009 IEEE Conference on Computer Vision and Pattern Recognition, Miami, FL, 2009, pp. 1070-1077.

[27] W. Yuan, R. Li, M. A. Srinivasan and E. H. Adelson, "Measurement of shear and slip with a GelSight tactile sensor," in 2015 IEEE International Conference on Robotics and Automation (ICRA), Seattle, WA, 2015, pp. 304-311.

[28] H. Yousef, M. Boukallel and K. Althoefer, "Tactile sensing for dexterous in-hand manipulation in robotics-A review," in Sensors and Actuators A: physical, 167(2): 171-187, 2011.

[29] X. Jia, R. Li, M. A. Srinivasan and E. H. Adelson, "Lump detection with a gelsight sensor," in 2013 World Haptics Conference (WHC), Daejeon, 2013, pp. 175-179.

[30] W. Yuan, Y. Mo, S. Wang and E. H. Adelson, "Active Clothing Material Perception Using Tactile Sensing and Deep Learning," in 2018 IEEE International Conference on Robotics and Automation (ICRA), Brisbane, QLD, 2018, pp. 4842-4849.

[31] W. Yuan, S. Dong, and E. H. Adelson, "Gelsight: High-resolution robot tactile sensors for estimating geometry and force," in Sensors 17.12 (2017): 2762, 2017.

[32] R. Li and E. H. Adelson, "Sensing and Recognizing Surface Textures Using a GelSight Sensor," in 2013 IEEE Conference on Computer Vision and Pattern Recognition, Portland, OR, 2013, pp. 1241-1247.

[33] Y. Noh et al., "A2-piece six-axis force/torque sensor capable of measuring loads applied to tools of complex shapes," in 2019 IEEE/RSJ International Conference on Intelligent Robots and Systems (IROS), Macau, China, 2019, pp. 7976-7981.

[34] L.L. Nathans, F.L. Oswald, K. Nimon, "Interpreting multiple linear regression: A guidebook of variable importance," in Pract. Assess. Res. Eval. vol.17, pp. 1-19, 2012.

[35] A.B. Dawood, H. Godaba and K. Althoefer, "Modelling of a Soft Sensor for Exteroception and Proprioception in a Pneumatically Actuated Soft Robot", in Annual Conference Towards Autonomous Robotic Systems 2019. Springer, Cham, 2019, pp. 99-110.

[36] R. D. Howe, "Tactile sensing and control of robotic manipulation", in Advanced Robotics, 8(3):245-261, 1993.

[37] T. Jack O., B. B. Sheil, and T. Sun, "Multi-axis force sensors: a state-of-the-art review," in Sensors and Actuators A: Physical (2020): $111772,2020$.

[38] M. Liu, Y. Zhao, Y. Shao, Q. Zhang and C. Liu, "3D Printed Force Sensor with Inkjet Printed Piezoresistive Based Strain Gauge," in 2018 IEEE SENSORS, New Delhi, 2018, pp. 1-4.

[39] L. Bohan, W. Xiaoliang, L. Shan, L. Junqin and Z. Guangshang, "Design and Analysis of a Novel Miniature Two-Axis Force Sensor," in 2019 2nd 
World Conference on Mechanical Engineering and Intelligent Manufacturing (WCMEIM), Shanghai, China, 2019, pp. 328-334.

[40] L. Xiong, G. Jiang, Y. Guo, H. Liu, "A three-dimensional Fiber bragg grating forcesensor for robot," in IEEE Sens. J. 18 (2018) 3632-3639.

[41] Minghua Luo, Xinhua Luo and Chunwei Pan, "A Stewart platform-based 3 -axis force sensor for robot fingers," in 2011 Second International Conference on Mechanic Automation and Control Engineering, Hohhot, 2011, pp. 37-40.

[42] O. Al-Mai, M. Ahmadi and J. Albert, "Design, Development and Calibration of a Lightweight, Compliant Six-Axis Optical Force/Torque Sensor," in IEEE Sensors Journal, vol. 18, no. 17, pp. 7005-7014, 2018.

[43] "ATI multi-axis force/torque sensors family," https://www.ati-ia.com/ products/ft/sensors.aspx

[44] "Hex force/torque sensor," https://onrobot.com/en/products/hex-6-axisforce-torque-sensor.

[45] G. Palli, L. Moriello, U. Scarcia, and C. Melchiorri, "Development of an optoelectronic 6-axis force/torque sensor for robotic applications," in Sensors and Actuators A: Physical,220:333-346, 2014.

[46] O. Al-Mai, M. Ahmadi and J. Albert, "A Compliant 3-Axis Fiber-Optic Force Sensor for Biomechanical Measurement," in IEEE Sensors Journal, vol. 17, no. 20, pp. 6549-6557, 2017.

[47] Y. Noh et al., "Image-Based Optical Miniaturized Three-Axis Force Sensor for Cardiac Catheterization," in IEEE Sensors Journal, vol. 16, no. 22, pp. 7924-7932, 2016.

[48] V. Duchaine, "Why tactile intelligence is the future of robotic grasping," in IEEE Spectrum: Blogs (2016).

[49] K. Shimonomura, "Tactile Image Sensors Employing Camera: A Review," in Sensors 19.18 (2019): 3933, 2019.

[50] C. Trueeb, S. Carmelo, and D. Raffaello, "Towards vision-based robotic skins: a data-driven, multi-camera tactile sensor," in arXiv preprint arXiv: 1910.14526 (2019).

[51] A. C. Abad and A. Ranasinghe, "Visuotactile Sensors With Emphasis on GelSight Sensor: A Review," in IEEE Sensors Journal, vol. 20, no. 14, pp. 7628-7638, 15 July15, 2020

[52] Russo, Stefania, et al. "Soft and stretchable sensor using biocompatible electrodes and liquid for medical applications," in Soft robotics 2.4 (2015): 146-154, 2015.

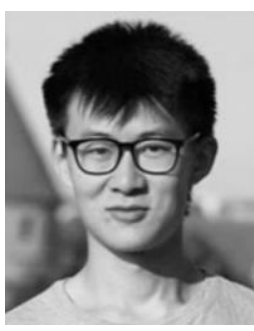

Wanlin Li received the B.Eng. degree in electronic engineering from Queen Mary University of London, United Kingdom, in 2016, where he is currently pursuing the Ph.D. degree in robotics. His current research interests include development of force and tactile sensors.

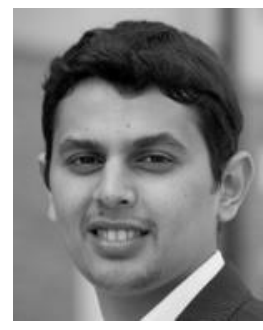

Akram Alomainy received the M.Eng. degree in communication engineering and the $\mathrm{Ph} . \mathrm{D}$. degree in electrical and electronic engineering (specialized in antennas and radio propagation) from Queen Mary University of London (QMUL), U.K., in July 2003 and July 2007, respectively. He joined the School of Electronic Engineering and Computer Science, QMUL, in 2007, where he is a Reader in Antennas \& Applied EM. His current research interests include small and compact antennas for wireless body area networks, radio propagation characterisation and modelling, antenna interactions with human body, computational electromagnetic, advanced antenna enhancement techniques for mobile and personal wireless communications, nano-scale networks and communications, $\mathrm{THz}$ material characterisation and communication links and advanced algorithm for smart and intelligent antenna and cognitive radio system. He is an elected member of UK URSI (International Union of Radio Science) panel to represent the UK interests of URSI Commission B (1 Sept 2014 until 31 Aug 2020).

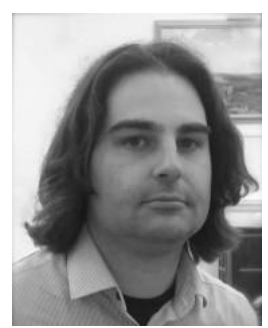

Ivan Vitanov received the $\mathrm{Ph} . \mathrm{D}$. degree in machine learning \& signal processing in 2016 and M.Sc. degree in scientific computing in 2007, both from Cranfield University, U.K. He also holds a B.Eng. Honours degree in electro-mechanical engineering from King's College London. Between 2007 and 2011 he was a Higher Research Scientist at BAE Systems' Advanced Technology Centre (ATC), U.K. He has previously held the position of Research Engineer at BMW's Research and Innovation Centre in Munich, Germany. He is currently a Research Associate with the Centre for Advanced Robotics (ARQ), Queen Mary, University of London. His current research interests are within the areas of autonomous robot navigation, motion planning and autonomous control, soft robotics for extreme environments, machine learning, sensor fusion and visuo-tactile sensing.

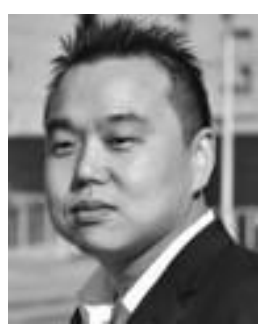

Yohan Noh received the B.S. degree from the Department of Mechanical Engineering, Seoul National University of Science and Technology, South Korea, in 2002, the B.S. degree from the Department of Electrical Engineering, Yonsei University, South Korea, in 2004, and the M.S. and Ph.D. degrees from the Department of Science and Engineering, Waseda University, Tokyo, Japan, in 2007 and 2011, respectively. He worked as a Research Associate for Robotics Research with the Department of Biomedical Engineering, King' s College London from 2013 to 2019. Since 2019, He has been currently a Lecturer with the Department of Mechanical and Aerospace Engineering, Brunel University London. His research interests include the development of force and tactile sensors, haptics, robot-assisted ultrasound diagnostic system, medical training system, and medical robots.

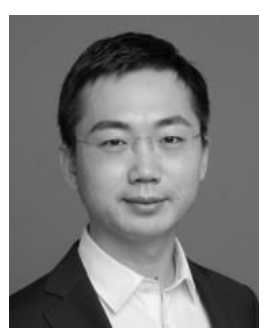

Peng Qi received the B.Eng. in Automation from Beijing Jiaotong University, Beijing, China, in 2010 and the M.S. degree in electrical engineering from KTH Royal Institute of Technology, Stockholm, Sweden, in 2012. He obtained the Ph.D. degree in Robotics from King's College London, United Kingdom, in Feb. 2016. He was a Research Fellow at the National University of Singapore from Sep. 2015 to Aug. 2016, and a visiting scholar (Honored) at Chinese University of Hong Kong from Sep. 2016 to Feb. 2017. He is currently an Assistant Professor in robotics at Tongji University since October 2016. Dr. Qi has been selected Shanghai Sailing Program and the "Chenguang Program", and involved in some research projects from the National Natural Science Foundation of China, etc. His current research interests include medical robotics, intelligent sensing and interaction, and autonomous intelligent system.

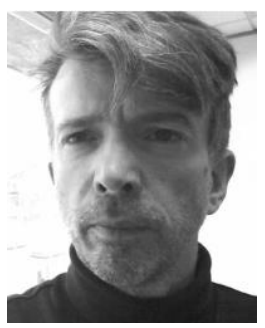

Kaspar Althoefer is an experienced roboticist leading competitively funded research on soft robotics, intelligent micro-sensing systems and interaction dynamics modelling with applications in minimally invasive surgery, assistive technologies and human-robot interaction at Queen Mary University of London. He acquired in excess of $£ 5.7 \mathrm{M}$ as Principal Investigator from national/international funding bodies and successfully completed $22 \mathrm{PhD}$ projects. Professor Althoefer's research team, currently comprising 10 postdoctoral research associates and $\mathrm{PhD}$ students, is involved in funded collaborative research with leading London hospitals, European research organisations and international companies creating novel robot-assisted solutions for cardiac catheterisation, foetal ultrasound monitoring, tissue diagnosis using miniaturised stiffness sensors and ergonomically-optimised human-robot interaction. Over the last decade, the team has built a large portfolio of projects in application-oriented research for the healthcare and manufacturing sectors with funding from organisations such as EPSRC, European Commission (including coordination of two EU-projects), Wellcome Trust and UK-based charities, exceeding $£ 30 \mathrm{M}$ and producing more than 250 peer-reviewed paper. 